\title{
A Method to Suppress Chest Compression Artifact Enhancing Capnography-Based Ventilation Guidance During Cardiopulmonary Resuscitation
}

\author{
Mikel Leturiondo ${ }^{1}$, JJ Gutiérrez ${ }^{1}$, Sofía Ruiz de Gauna ${ }^{1}$, Jesus Ruiz ${ }^{1}$, Luis A Leturiondo ${ }^{1}$, James K \\ Russell $^{2}$, Mohamud Daya ${ }^{2}$ \\ ${ }^{1}$ University of the Basque Country (UPV/EHU), Bilbao, Spain \\ ${ }^{2}$ Oregon Health \& Science University (OHSU), Portland OR, USA
}

\begin{abstract}
Capnography-based ventilation rate guidance is valuable and widely used by advanced life support during cardiopulmonary resuscitation (CPR). However, there is a high incidence of induced chest compression (CC) oscillations that decreases the reliability of automated ventilation detection. We used 30 out-of-hospital cardiac arrest episodes containing the capnogram and transthoracic impedance signals. The algorithm detects the presence of distorted ventilations in the capnogram. It calculates the artifact envelope during the alveolar plateau and removes the artifact during capnogram baseline, thus obtaining a non-distorted waveform. The goodness of the method was assessed by comparing the performance of a ventilation detection algorithm before and after artifact suppression. From a total of 6387 annotated ventilations, $34 \%$ of them were classified as distorted. Global sensitivity and positive predictive value (Se/PPV, \%) improved from 77.9/74.0 to 97.0/95.8. Median value of the unsigned error (\%) of the estimated ventilation rate decreased from 19.6 to 4.5 and the accuracy for detection of over-ventilation increased with cleaned capnograms. Capnogram-based ventilation guidance during $C P R$ was enhanced after $C C$ artifact suppression. Our method preserved the tracing of $\mathrm{CO}_{2}$ concentration caused by ventilations, allowing other clinical uses of the capnography during resuscitation.
\end{abstract}

\section{Introduction}

During out-of-hospital cardiac arrest (OHCA) episodes, advanced life support (ALS) treatment usually involves advanced airway placement, high-quality cardiopulmonary resuscitation (CPR) and early defibrillation [1,2].

Guidance on ventilation rate is key during CPR, thus, capnography is widely used by the ALS, but for this purpose, a clinically interpretable waveform where the different phases of the respiratory cycle must be clearly identifiable. Several studies have reported the appearance of fast oscillations synchronized with provided chest compressions (CCs) superimposed on the capnogram, often completely distorting the normal tracing $[3,4]$.

Filtering approaches have been widely proposed to remove chest compression artifact from the electrocardiogram during CPR [5]. In a recent study, we proposed a few filtering techniques to remove those oscillations from the capnogram [6]. All of them improved the automated detection of ventilations. However, these frequency domain techniques present an important limitation where the artifact appears. Resulting capnogram waveform represents only the average of the original $\mathrm{CO}_{2}$ concentration. Thus, filtered capnograms are not suitable for clinical analysis.

In this study, we explored an alternative method to suppress chest compression oscillations from the capnography waveform. Our proposal is based on a time domain analysis, and was not only designed to improve ventilation detection, but also to provide a capnogram that resembles the "real" tracing caused by ventilations.

\section{Materials and methods}

\subsection{Database description and annotation}

The dataset analyzed in this study was a subset (30 episodes) of highly distorted capnogram waveforms extracted from a large OHCA registry collected by Tualatin Valley Fire \& Rescue (TVF\&R), an advanced life support (ALS) Emergency Medical Services (EMS) agency, between 2011 and 2016. Episodes were recorded using Heartstart MRx monitor-defibrillators (Philips USA) equipped with real-time CPR feedback (QCPR, Laerdal Medical, Norway) and sidestream capnography (Microstream, Oridion Systems Ltd, Israel) to provide a High-Quality CPR. For each episode, we extracted two 
concurrent signals: the transthoracic impedance (TI) signal, acquired from the defibrillation pads and the capnogram, acquired using sidestream technology.

Ventilation annotations from a previous study were used [4]. In that study, episodes with more than $1 \mathrm{~min}$ of evident CC artifact were classified as distorted. Distorted episodes were then grouped into type I, type II and type III (highly distorted) artifact categories. Ventilations were annotated using the raw TI signal as reference. TI signal was low-pass filtered to remove oscillations caused by CC. Thus, enhancing slow fluctuations caused by ventilations. All processed TI signals were visually examined to manually annotate the position of each ventilation. Resulting highly distorted episodes and ventilation annotations were used in the present study as our gold standard to prove the reliability of the artifact suppression method. Additionally, in this study we also classified each capnogram cycle (ventilation) as distorted or non-distorted by $\mathrm{CC}$ artifact following the pattern shown in Figure 1.

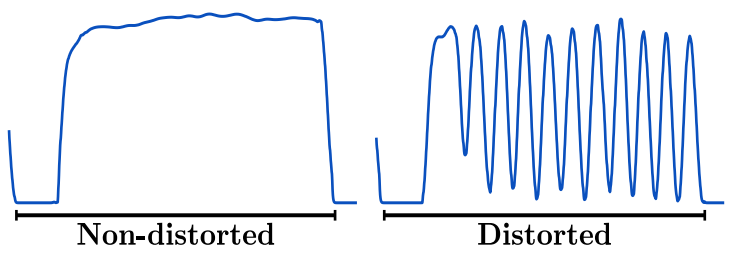

Figure 1. Capnography signal segments.

\subsection{Chest compression artifact suppression}

Following the hypothesis that the real $\mathrm{CO}_{2}$ concentration produced by ventilations remains in the envelope of the induced CC artifact, we designed a method that restores the "real" tracing of the capnography waveform. A graphical explanation of the method performance is given in Figure 2, where a segment of a distorted capnogram (blue line) is depicted with the resulting capnogram after applying the method (dotted red line).

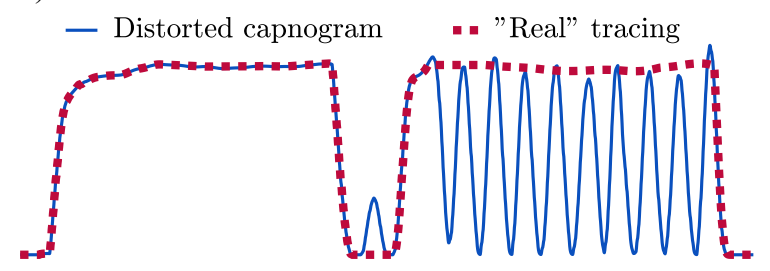

Figure 2. Graphical explanation of the artifact suppression method.

The artifact suppression algorithm operates in real-time, with one second buffered delay, to process, detect and fix the capnography waveform. Due to artifact origin and type variety [4], the suppression method divides its operation into low and high $\mathrm{CO}_{2}$ concentration intervals. This division helps to determine how to extract the envelope of the capnography waveform.
The functioning of the algorithm can be divided into three main steps:

- Feature computation: The algorithm applies two thresholds to distinguish between low $\mathrm{CO}_{2}$ concentration phase (Figure 3, grey line) and high $\mathrm{CO}_{2}$ concentration phase (Figure 3, blue line), $T h_{\mathrm{lo}}$ and $T h_{\mathrm{hi}}$ respectively. This thresholds determine the beginning instances of each interval $\left(t_{\mathrm{lo}}^{i}\right.$ and $\left.t_{\mathrm{hi}}^{i}\right)$. For every capnogram fluctuation, both thresholds are updated using the last high $\mathrm{CO}_{2}$ value (red diamond). Finally, every capnogram fluctuation is characterized by two features:

$$
\begin{array}{ll}
- & D_{\mathrm{lo}}: \text { Low } \mathrm{CO}_{2} \text { interval duration, } D_{\mathrm{lo}}=t_{\mathrm{hi}}^{i}-t_{\mathrm{lo}}^{i} \\
- & D_{\mathrm{hi}}: \text { High } \mathrm{CO}_{2} \text { interval duration, } D_{\mathrm{hi}}=t_{\mathrm{lo}}^{i+1}-t_{\mathrm{hi}}^{i}
\end{array}
$$

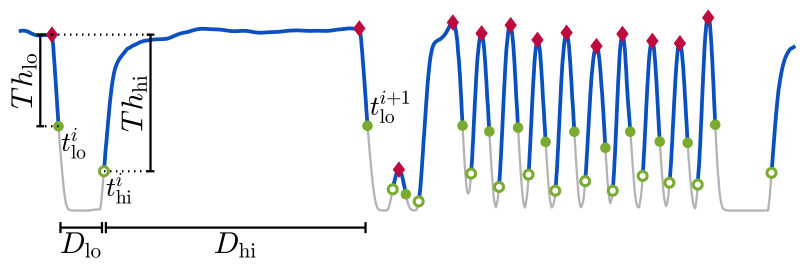

Figure 3. Example of feature computation.

- Artifact detection and reconstruction: The operation of the method depends on which interval is analyzing. During a low $\mathrm{CO}_{2}$ interval, the algorithm analyzes the following $\mathrm{CO}_{2}$ interval and vice versa, as shown in Figure 4 . The algorithm detects the presence of artifact when:

- During low $\mathrm{CO}_{2}$ interval (Figure 4, left panel), the following high $\mathrm{CO}_{2}$ interval duration $\left(D_{\mathrm{hi}}\right)$ is less than or equal to a high interval duration threshold, $D t h_{\mathrm{hi}}$.

- During high $\mathrm{CO}_{2}$ interval (Figure 4, right panel), the following low $\mathrm{CO}_{2}$ interval duration $\left(D_{\mathrm{lo}}\right)$ is less than or equal to a low interval duration threshold, $D t h_{\mathrm{lo}}$.

Only in presence of artifact, the algorithm extracts the local minima (upward arrowheads) and local maxima (downward arrowheads) of the oscillations, during low and high intervals respectively. Finally, it connects these points with a linear interpolation obtaining the lower and upper envelopes.
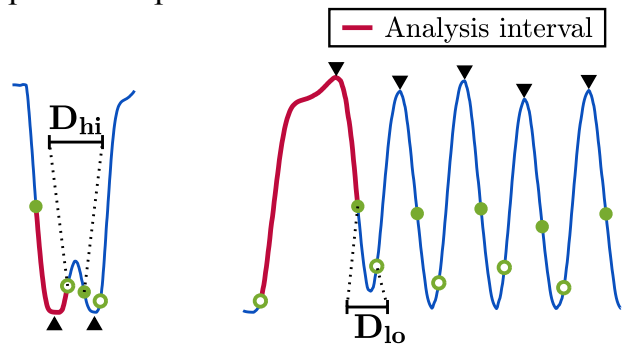

Figure 4. Detection of chest compression oscillations. 
- Envelope smoothing: Additionally a sliding window average filter (i.e. moving average) is applied only to smooth the upper envelope, as shown in Figure 5.
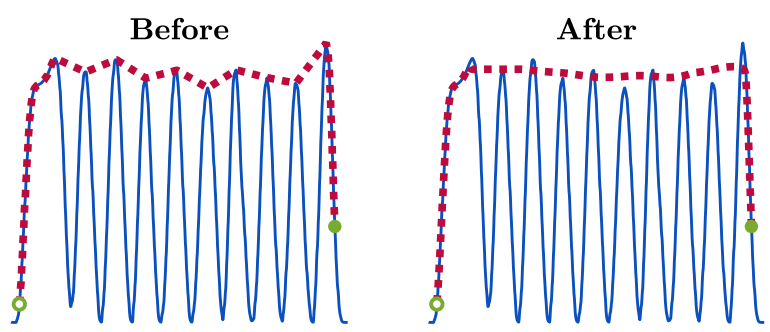

Figure 5. Upper envelope moving average smoothing.

\subsection{Performance evaluation}

We computed the effectiveness of the artifact suppression method in terms of the sensitivity (Se) and positive predictive value (PPV) of an automated ventilation detection algorithm before and after artifact suppression. Se values were defined as the proportion of correctly detected ventilations among all annotated ventilations. PPV values were defined as the proportion of correctly detected ventilations among all detections.

We also evaluated the performance of the method in the estimation of ventilation rate; we computed, for each episode, the number of ventilations given in the las minute updated every $10 \mathrm{~s}$. Ventilation rate measurements obtained before and after applying the artifact suppression method were compared with the measurements computed form the gold standard by calculating the unsigned percent error.

Finally, accuracy in the detection of excessive ventilation rates was also tested. According to the general recommendation in current resuscitation guidelines, ventilation rates above $10 \mathrm{~min}^{-1}$ during CPR can be considered as excessive $[1,2]$. In this case, Se was defined as the proportion of annotated alarms that were given by the algorithm, and PPV as the proportion of given alarms that were indeed annotated.

\section{Results}

From the 30 OHCA episodes that were analyzed, with a mean duration of $34( \pm 18)$ minutes, a total of 6387 ventilations were annotated, with a mean of $213( \pm 92)$ ventilations per episode. From these, $4215(66 \%)$ corresponded to non-distorted ventilations and 2172 (34\%) to distorted ventilations.

The performance of the automated ventilation detection algorithm was computed before and after applying the suppression method. For the whole set, global Se/PPV improved from $77.9 \% / 74.0 \%$ to $97.0 \% / 95.8 \%$. Figure $6 \mathrm{~A}$ illustrates the distribution of Se and PPV per episode given by the ventilation detection algorithm. Overall, both medians increased and dispersion in Se and PPV was also reduced. Se/PPV IQR decreased from $20.1 \% / 29.2 \%$ to $1.6 \% / 3.8 \%$.
(A)

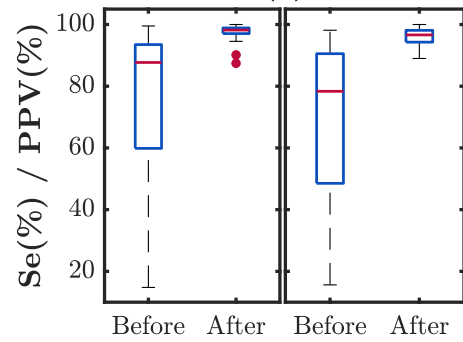

(B)

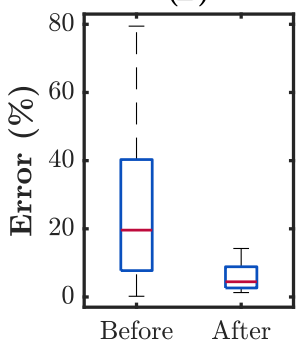

Figure 6. A) Distribution of Se and PPV per episode.

B) Distribution of the unsigned error in percentage per episode in the estimation of ventilation rate.

Figure 6B depicts through box plots the distribution of the unsigned error in percentage between the estimation and the gold-standard ventilation rate value. As is the case with $\mathrm{Se} / \mathrm{PPV}$, the median error noticeably decreases from $19.6 \%$ to $4.5 \%$ and the IQR from $32.6 \%$ to $6.3 \%$.

The influence of the artifact suppression in the detection of excessive ventilation rates was also computed. Globally, Se/PPV were $95.5 \% / 71.9 \%$ before and $98.7 \% / 93.5 \%$ after artifact suppression. In this case the improvement was significantly higher for PPV values with a slightly increase in Se. An increase in PPV implied a reduction in false overventilation alarms.

Finally, Figure 7 visually illustrates the performance of the method. The raw capnogram is depicted in blue and the resulting artifact suppression waveform in red, superimposed to the raw capnogram.

a)

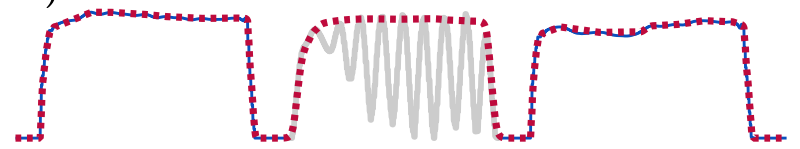

b)
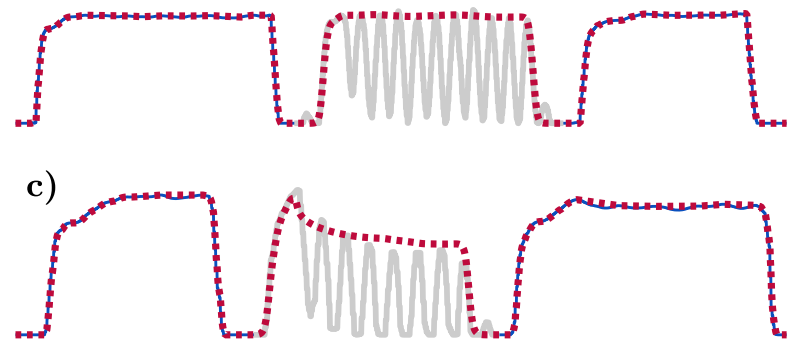

Figure 7. Examples of restored capnograms. 


\section{Discussion}

During OHCA resuscitation episodes a high incidence of induced oscillations has been reported. Induced CC artifact on OHCA capnograms negatively affects the automated detection of ventilations. This means that real time monitoring of ventilation rate could become unreliable in some circumstances. Filtering approaches have been designed to reduce the impact of this problem: classic low pass filtering, with either fixed or adaptive schemas, improved ventilation detection accuracy. However, the resulting capnogram did not accurately represent the "real" tracing caused by ventilations, limiting the application just to improve the automated detection of ventilations.

Visual tracking of ventilation instances and the analysis of the evolution of $\mathrm{ETCO} 2$ values during CPR may be interfered by the presence of induced CC oscillations superimposed on capnograms. In this study, we proposed an artifact suppression method that extracts the envelope of the chest compression oscillations. It has been demonstrated that the resulting artifact suppression output resembles the $\mathrm{CO}_{2}$ fluctuations caused by ventilations. We also validated the improvement in the automated detection of ventilations, real-time ventilation rate estimation and detection of excessive ventilation rates. Unfortunately, we could not numerically assess the goodness of the resulting capnogram waveform and the error committed. Thus, we visually analyzed capnogram segments showing adjacent distorted and non-distorted ventilations (Figure 5). In most of the cases, the envelope of the distorted capnogram preserves the $\mathrm{CO}_{2}$ tracing of preceding and following nondistorted ventilations (Figure 5A,B). Only a few of them did not follow the pattern of adjacent non-distorted respiratory cycles (Figure 5C).

\section{Conclusions}

Capnogram-based ventilation guidance during CPR was enhanced after $\mathrm{CC}$ artifact suppression. Our method preserved the tracing of $\mathrm{CO} 2$ concentration caused by ventilations, allowing other clinical uses of capnography during resuscitation.

\section{Acknowledgements}

This work received financial support from the Basque Government (Basque Country, Spain) through the project IT1087-16 and the predoctoral research grant PRE-20172-0201. The authors thank the TVF\&R EMS providers for collecting the data used in this study.

\section{References}

[1] Soar J, Nolan JP, Böttiger BW, et al. European Resuscitation Council guidelines for resuscitation 2015. Section 3. Adult advanced life support. Resuscitation 2015;95:100-147.

[2] Link MS, Berkow LC, Kudenchuk PJ, et al. 2015 American Heart Association Guidelines Update for Cardiopulmonary Resuscitation and Emergency Cardiovascular Care. Part 7: adult advanced cardiovascular life support. Circulation 2015;132(18 suppl 2):S444-S464.

[3] Idris AH, Daya M, Owens P, et al. High incidence of chest compression oscillations associated with capnography during out-of-hospital cardiopulmonary resuscitation. Circulation 2010;122:A83.

[4] Leturiondo M, Ruiz de Gauna S, Ruiz JM, et al. Influence of chest compression artifact on capnogram-based ventilation detection during out-of-hospital cardiopulmonary resuscitation. Resuscitation 2018;124:63-68.

[5] Gong Y, Chen B, Li Y. A review of the performance of artifact filtering algorithms for cardiopulmonary resuscitation. Journal of Healthcare Engineering 2013;4(2):185-202.

[6] Gutierrez JJ, Leturiondo M, Ruiz de Gauna S, et al. Enhancing ventilation detection during cardiopulmonary resuscitation by filtering chest compression artifact from the capnography caveform. PLOS ONE 2018; 13(8):e0201565.

Address for correspondence.

Mikel Leturiondo Sota

mikel.leturiondo@ehu.eus

School of Engineering

Plaza Torres Quevedo, 1, 48013-Bilbao (Spain) 